\title{
Medico-legal Implications of Changes in Handwriting of Some Hemodialysis Egyptian Patients (A Prospective Study)
}

\author{
Hala A. Mohamed ${ }^{1}$, Magdy EISharkawy ${ }^{2}$ and Fatma E.Agha ${ }^{3}$
}

${ }^{1}$ Forensic Department ministry of Justice, Egypt

${ }^{2}$ Nephrology Department, Faculty of Medicine, Ain-Shams University, Cairo, Egypt.

${ }^{3}$ Forensic Medicine and Clinical Toxicology Department, Faculty of Medicine for Girls, AI-Azhar University, C a i r o, Egypt.

\begin{abstract}
Little attention has been paid to the possibility of cognitive deficits in patients with major organs failure such as the liver, kidney, or heart. Hemodialysis patients are at high risk for cognitive dysfunction, psychometric deficits and metabolic alternations than chronic kidney disease alone that can affect the quality of hand writing and impair its proper recognition. Aim of the study it aims to : Structurally analyze changes in handwriting characteristics among some hemodialysis Egyptian patients and to assess their medico-legal implications of the handwriting. Thirty seven stable hemodialysis patients were included in this study. All the patients were asked to write a sample. Typing tool, writing surface, writing mode and time for filling the form were standardized for all the patients. Qualitative data were presented as frequencies and percentages. Results: Handwriting analysis showed, There was a statistically significant difference $(P<0.001)$ regarding Writing speed (slow speed (81.1\%), medium (13.5\%), and very slow speed (5.4\%), Pen pressure grades (light pressure (62.2\%), heavy pressure (24.3\%) and medium pressure $(13.5 \% »$, word size (large word size $(46 \%)$, medium word size $(40.5 \%)$ and small word size (13.5\%), Interrupted stroke (97.3\%), Curved (zigzag) stroke (100\%), Extra stroke (100\%) and Stiff stroke(100\%), dirtiness(78.4\%), spacing represented (97.3\%)over-Writing (73\%) defined as number of over-writings done by each subject / total number of words written by each subject $\mathrm{x}$ 100. Conclusion: hemodialysis patients had a characteristic impairment in handwriting skills which may indicate cognitive deficit that cannot be elicited by clinical examination and investigations. These changes may carry great medico legal implications, and should be considered when analyzing their hand writings.
\end{abstract}

Keywords Uremic encephalopathy; End stage chronic renal failure, Hemodialysis, Handwriting.

\begin{abstract}
Introduction
Handwriting is a motor task which requires extensive cognitive processing as each stroke is planned and executed. ${ }^{1}$ On the other hand, diseases have an effect on person's handwriting; this fact has been established since the principles of handwriting identification were first recognized. ${ }^{2}$ In the medical field, it can be used as an aid in diagnosis and tracking of diseases like Parkinson's disease, Huntingdon's Disease, Alzheimer's disease, and even cancer. ${ }^{3}$ Since handwriting constitutes the tracing of neuromuscular coordination, the microscopic characteristics of handwriting strokes
\end{abstract}

become an important method for examining human neuromuscular development. ${ }^{4 \& 5}$ To a lesser degree stress, alcoholism, and drug abuse have also been researched. ${ }^{6} \& 7$

Kidney failure is one of the leading causes of disability and death. Neurological complications of uremia, like uremic encephalopathy, atherosclerosis, neuropathy and myopathy fail to fully respond to dialysis. Encephalopathy is often associated with headache, visual abnormalities, tremor, asterixis, multifocal myoclonus, chorea and seizures. These signs fluctuate from day to 
day or sometimes from hour to hour. ${ }^{8}$ Uremic encephalopathy which is an organic brain disorder, it develops in patients with acute or chronic renal failure, usually when creatinine clearance levels fall and remain below $15 \mathrm{~mL} / \mathrm{min} .{ }^{9}$ Dialysis encephalopathy is a sub-acute, progressive and often fatal disease that presents with dysarthria, dysphasia, apraxia, personality changes, psychosis, myoclonus, seizures and finally dementia. ${ }^{10 \& 11}$ Dementia is more common in patients with renal failure than in the general population. The incidence of dementia in aged patients undergoing dialysis is estimated at $4.2 \% .^{12}$ Cerebral atrophy is common in patients with chronic renal failure, even in patients without evident cognitive, affective or behavioral changes. $^{13}$

Movement disorders in patients with renal failure can occur as a result of encephalopathy, medication or structural lesions. Several types of involuntary movements can occur in metabolic encephalopathy. Asterixis or flapping tremor "is probably caused by sudden loss of tonus, originating from cortical dysfunction. ${ }^{14 \& 15}$ In uremic myoclonus the patient exhibits shock- like contractions of a muscle or a group of muscles, these contractions are irregular in rhythm and amplitude and are followed by relaxation. ${ }^{16}$ Uremic "twitch convulsive" syndrome is a typical movement disorder in uremic encephalopathy that consists of intense asterixis and myoclonic jerks that are accompanied by fasciculations, muscle twitches and seizures. ${ }^{10 \& 17}$

Uremic proximal myopathy is a common finding in patients with end stage renal disease, appears in patients with a glomerular filtration rate less than $25 \mathrm{~mL} / \mathrm{min}$ and the progression parallels the decline of renal function. ${ }^{18}$ Haemodialysis patients are reported to face psychosocial problems like depression, anxiety, suicide and delirium, which can contribute to conflictions between themselves and their medical carers. Such findings could be attributed in part to the stressful conditions in the haemodialysis treatment modality, including frequent visits and prolonged waiting time in the dialysis unit. ${ }^{19}$ Hemodialysis patients are at high risk for cognitive dysfunction, psychometric deficits and metabolic alternations than chronic kidney disease alone that can affect the quality of hand writing and impair its proper recognition. ${ }^{20}$ No recordable scientific studies have been found to show handwriting impairment in cases of end stage chronic renal failure with haemodialysis, so, this is the first study to structurally analyze changes in handwriting characteristics among some hemodialysis Egyptian patients.

\section{Subject and Methods Subjects}

Thirty seven patients suffering from end stage chronic renal failure on regular hemodialysis three sessions / week (26 males and 11 females), ages ranged from 20-70 years attending the Dialysis Unite of Nasser institute and private dialysis center in Cairo within two years were included in this study. Prior to inclusion, each patient underwent a comprehensive renal investigation and gave a full valid consent. At the time of testing all patients had stabilized general condition, adult age with at least primary educational level.

The following exclusion criteria were applied in this study:

a) Administration of psychotropic substances.

b) Known major neuro-psychiatric disorder and alcohol abstinence.

c) patients suffering from other systemic diseases such as heart disease and any type of cancer.

d) Illiterate were excluded from the study.

An approval for this research work was obtained from The Ethics Committee.

\section{Methods}

Handwriting samples were written in exemplar by participants themselves according to the Arabian model. Participants were asked to write their name, job, address, ID number, date of birth, monthly income, level of education numbers of family and living rooms, history of previous diseases and their opinion in the level of hospital medical services and treatment. In all cases, the original documents related to the studied patients before the start of hemodialysis were obtained. All subjects used the same written papers, pads, and pens which facilitates estimation of the fine changes in handwriting features.

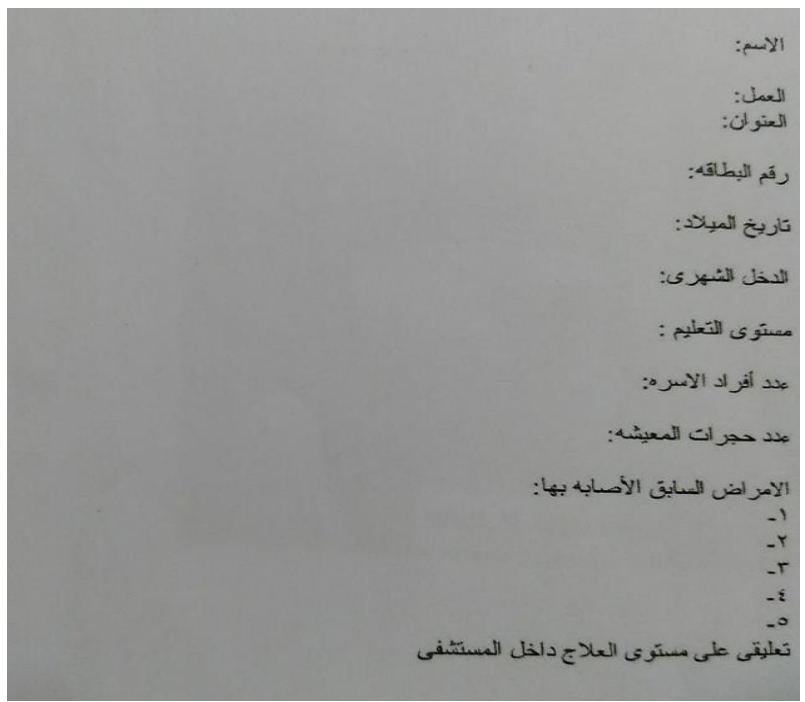

Photograph(1). A model of the request handwriting All handwriting samples and exemplars were secured in an anonymous envelop which in opened only for analysis. 


\section{Ethical Considerations}

To secure the confidentiality of the participants the obtained documents were kept anonymously throughout the study.

\section{Handwriting analysis}

Writing samples were taken, and a letter by letter detailed analysis by experts of handwriting in Ministry of Justice-Egypt using magnified lenses and different microscopes. Every mark on the script or unusual movement in the trace was noted during the analysis and recorded individually on hard copy spreadsheets for each participant; further the frequency of every letter written was totaled and analyzed alongside the main data. Various writing traits were analyzed including writing speed, pen pressure grades, word size, interrupted strokes, curved stroke, extra stroke, stiff stroke, dirtiness, spacing ,overwriting. ${ }^{21}$

\section{Statistical Analysis}

Statistical analysis of data was performed with PASW Statistics 18.0 (Predictive Analytics Software) for Windows. Qualitative data were presented as frequencies and percentages. Chi-square $\mathrm{X}^{2}$ test was used to compare between qualitative data. Quantitative data were presented as minimum, maximum, mean and standard deviation values. The significance level was set at $\mathrm{P} \leq 0.05$.

\section{Results \\ Demographic analysis}

The percentage of male cases predominate female cases $(70.3 \%$ and $29.7 \%)$ respectively fig(1), the distribution of age categories was represented in fig(2). While educational level of patients was demonstrated in table(1) and fig (3).

\section{Handwriting analysis}

In regard to writing speed, there was a statistically significant difference

$(P<0.001)$ among patients under study table (2) and fig (4). The speed of handwriting was classified as follows:-

Very slow, represented the lowest percentage among the studied subjects (5.4\%).

Slow speed, which may be intended resulting into writing similar to drawing it showed the highest percentage of the studied sample (81.1\%).

Medium speed, coinciding with the natural or usual speed of handwriting, represented (13.5\%).

Table(3) and Figure(5) showed pen pressure grade in the studied sample, there was a statistically significant difference $(P<0.001)$ between the different pen pressure grades. It was classified as follows:-

Heavy pressure: the thicker strokes and heavy indentation marks could be felt on the back of the paper which represented (24.3).

Medium pressure: medium thickness of strokes and heavy indentations could be felt on the back of the paper; it showed the lowest percentage among the studied subjects (24.3).
Light pressure on the back of the paper which showed the highest prevalence among the studied subjects (62.2\%).

In the present study, there was a statistically significant difference $(P<0.05)$ between the different word size grades. Subjects with large word size showed the highest prevalence (46\%) followed by subjects with medium size word (40.5). while subjects with small word size showed the least prevalence (13.5\%) table(4) and figure(6).

Handwriting for hemodialysis patients were compared to their old documents and there were a statistically significant difference $(P<0.001)$ regarding handwriting speed, word size, pen pressure, strokes, word spacing, dirtiness and overwriting.

Handwriting strokes data were presented as number of strokes done by each subject / total number of words written by each subject $\mathrm{x} 100$, so they will be presented as mean \% of strokes as follows table(5) and figure(7):-

Thirty-six subjects (97.3\%) of the studied sample showed interrupted stroke in their handwriting. The mean and standard deviation values of interrupted stroke in the studied sample were $3.9 \pm 2.5 \%$ with a minimum of $0.5 \%$ and a maximum of $11.1 \%$.

- Thirty-seven subjects (100\%) of the studied sample showed semi-tremors strokes (curved strokes) in their handwriting. The mean and standard deviation values of curved strokes in the studied sample were $7.8 \pm 5.4 \%$ with a minimum of $1.2 \%$ and a maximum of $27.5 \%$.

Thirty-seven subjects (100\%) of the studied sample showed rigid strokes (stiff s t r o ke s) in their handwriting. The mean and standard deviation values of stiff strokes in the studied sample were $5.4 \pm 4.3 \%$ with a minimum of 1.3 $\%$ and a maximum of $25.9 \%$.

In the present study, the dirtiness and overwriting data were presented as number of dirt and overwriting done by each subject / total number of words written by each subject $\mathrm{x} 100$, so they will be presented as mean \% of dirtiness and overwriting table(6) and figure (7) as follows:

Twenty-nine subjects' (78.4\%) of the studied sample showed dirtiness in their handwriting. The mean and standard deviation values of dirtiness in the studied sample were 4.1 $\pm \quad 1.7 \%$ with a minimum of $0.3 \%$ and a maximum of $6.8 \%$.

Thirty-six subjects (97.3\%) of the studied sample showed inter letters and inter word spacing in their handwriting. The mean and standard deviation values of separation in the studied sample were $1.8 \pm 0.5 \%$ with a minimum of $0.3 \%$ and a maximum of $2.4 \%$.

Twenty-seven subjects (73\%) of the studied sample showed over-writing in their handwriting. The mean and standard deviation values of over- writing in the sample were $1.5 \pm$ 
$0.7 \%$ with a minimum of $0.3 \%$ and a maximum of $2.8 \%$.

It had also been remarked other characteristics in some patients although, these are not repetitive in all patients, but they are of great importance, such as aberration, interference of lines and alignment (ascending and descending).
Five subjects (13.5\%) of the studied samples showed aberration in their handwriting.

Two subjects (5.4\%) of the studied samples showed interference of lines in their handwriting .

Ten subjects (27.03\%) of the studied samples showed alignment of handwriting (ascending and descending).

Table (1): Frequency a n d percentage of educational 1 e $\mathrm{v}$ e $\mathrm{l}$ in the studied sample (n=37).

\begin{tabular}{|c|c|c|}
\hline Educational level & Frequency(\%) \\
\hline Postgraduate degree & 1 & 2.7 \\
\hline University (High) & 11 & 29.7 \\
\hline Medium & 17 & 45.9 \\
\hline Below medium & 8 & 21.6 \\
\hline
\end{tabular}

Table (2): Chi-square analys is $X^{2}$ of handwriting speed grade among the studied patients with chronic renal failure and hemodialysis $(n=37)$

\begin{tabular}{|c|c|c|c|c|}
\hline Speed & Frequency & $\%$ & $\mathrm{X}^{2}$ value & P-value \\
\hline Very slow & 2 & 5.4 & \multirow{3}{*}{36.500} & \multirow{3}{*}{$<0.001^{*}$} \\
\hline Slow & 30 & 81.1 & & \\
\hline Medium & 5 & 13.5 & & \\
\hline
\end{tabular}

*: Significant at $P \leq 0.05$

Table (3): Chi-square analys is $X^{2}$ of pen pressure grade among the studied patients with chronic renal failure and hemodialysis ( $\mathbf{n}=37)$.

\begin{tabular}{|c|c|c|c|c|}
\hline Pen pressure & Frequency & $\%$ & $X^{2}$ value & P-value \\
\hline Heavy & 9 & 24.3 & \multirow{3}{*}{14.486} & \multirow{2}{*}{$0.001^{*}$} \\
\cline { 1 - 3 } Medium & 5 & 13.5 & \multirow{2}{*}{14.486} & \\
\hline Light & 23 & 62.2 & & \\
\hline
\end{tabular}

*: Significant at $P \leq 0.05$

Table (4): Chi-square analys is $X^{2}$ of word size grade in among the studied patients with chronic renal failure and hemodialysis $(n=37)$.

\begin{tabular}{|c|c|c|c|c|}
\hline Word size & Frequency & $\mathbf{\%}$ & $\mathbf{X}^{\mathbf{2}}$ value & P-value \\
\hline Small & 5 & 13.5 & & \multirow{2}{*}{6.703} \\
\cline { 1 - 3 } Medium & 15 & 40.5 & $0.05^{*}$ \\
\hline Large & 17 & 46 & & \\
\hline
\end{tabular}

*: Significant a t $P \leq 0.05$

Table (5): Mean, minimum and maximums \% of different types of handwriting stroke among the studied patients with chronic renal failure and hemodialysis $(n=37)$

\begin{tabular}{|c|c|c|c|}
\hline Types of strokes & Mean\% $\%$ SD & Minimum\% & Maximum \% \\
\hline Interrupted stroke & $3.9 \pm 2.5$ & 0.5 & 11.1 \\
\hline Curved (zigzag)stroke & $7.8 \pm 5.4$ & 1.2 & 27.5 \\
\hline Extra stroke & $5.8 \pm 4.2$ & 0.5 & 17.5 \\
\hline Stiff stroke & $5.4 \pm 4.3$ & 1.3 & 25.9 \\
\hline
\end{tabular}

Table (6): Mean, minimum and maximum \% of dirtiness and overwriting among the studied patients with chronic renal failure and hemodialysis $(n=37)$.

\begin{tabular}{|c|c|c|c|c|}
\hline Handwriting parameters & No. of patients & Mean \% \pm SD & Minimum\% & Maximum\% \\
\hline Dirtiness & 29(78.4\%) & $4.1 \pm 1.7$ & 0.3 & 6.8 \\
\hline Inter letters and inter word spacing & $36(97.3 \%)$ & $1.8 \pm 0.5$ & 0.3 & 2.4 \\
\hline Over-writing & $27(73 \%)$ & $1.5 \pm 0.7$ & 0.3 & 2.8 \\
\hline
\end{tabular}




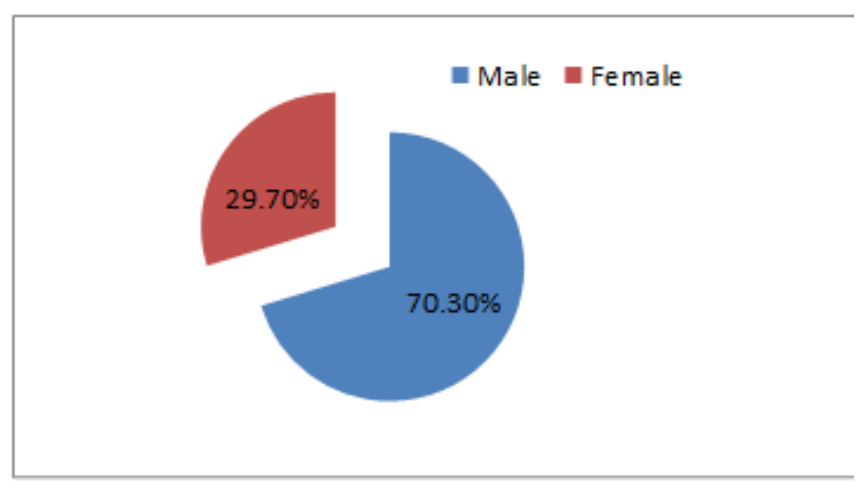

Figure (1):Pie chart shows gender distribution of the studied patients with chronic renal failure and hemodialysis $(\mathbf{n}=37)$.

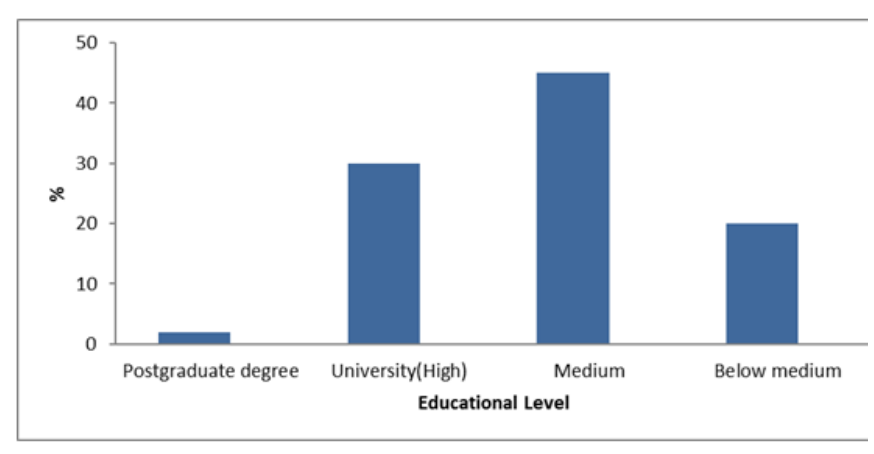

Figure (3): Column chart shows the educational levels among the studied patients with chronic renal failure and hemodialysis $(\mathbf{n}=37)$.

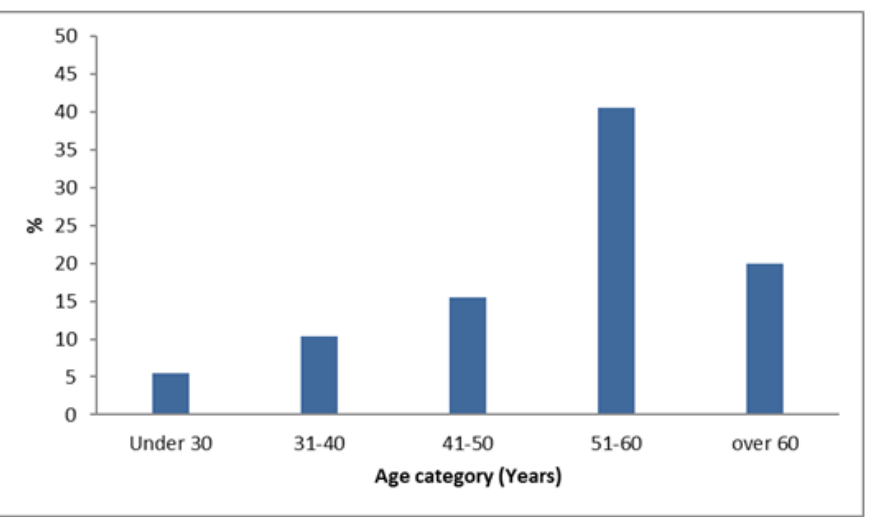

Figure (2): Column chart shows age categories among the studied patients with chronic renal failure and hemodialysis $(n=37)$.

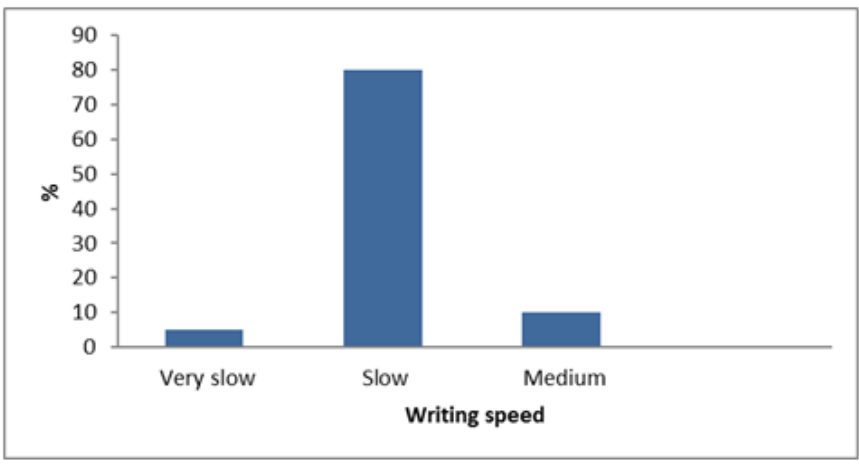

Figure (4): Column chart shows writing speed among the studied patients with chronic renal failure and hemodialysis $(n=37)$.

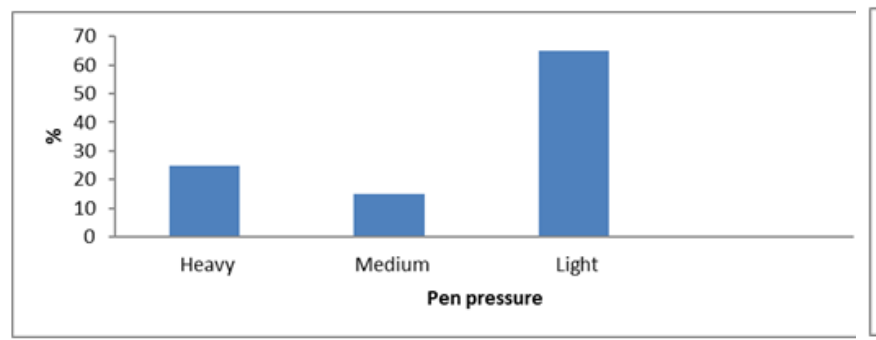

Figure (5):Column chart shows pen pressure among the studied patients with chronic renal failure and hemodialysis $(n=37)$.

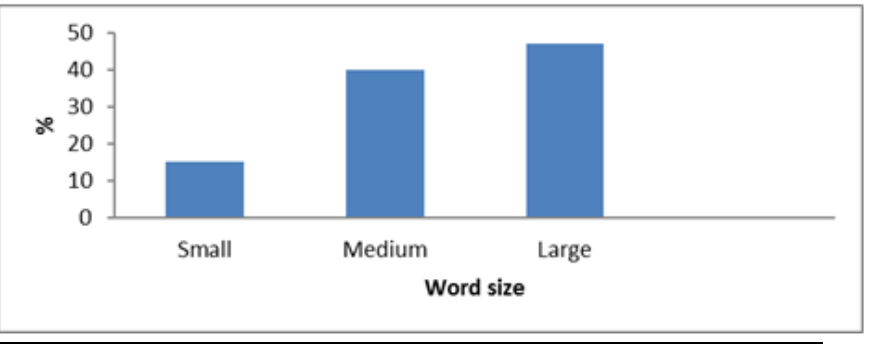

Figure (6): Column chart shows word size among the studied patients with chronic renal failure and hemodialysis ( $n=37)$. 


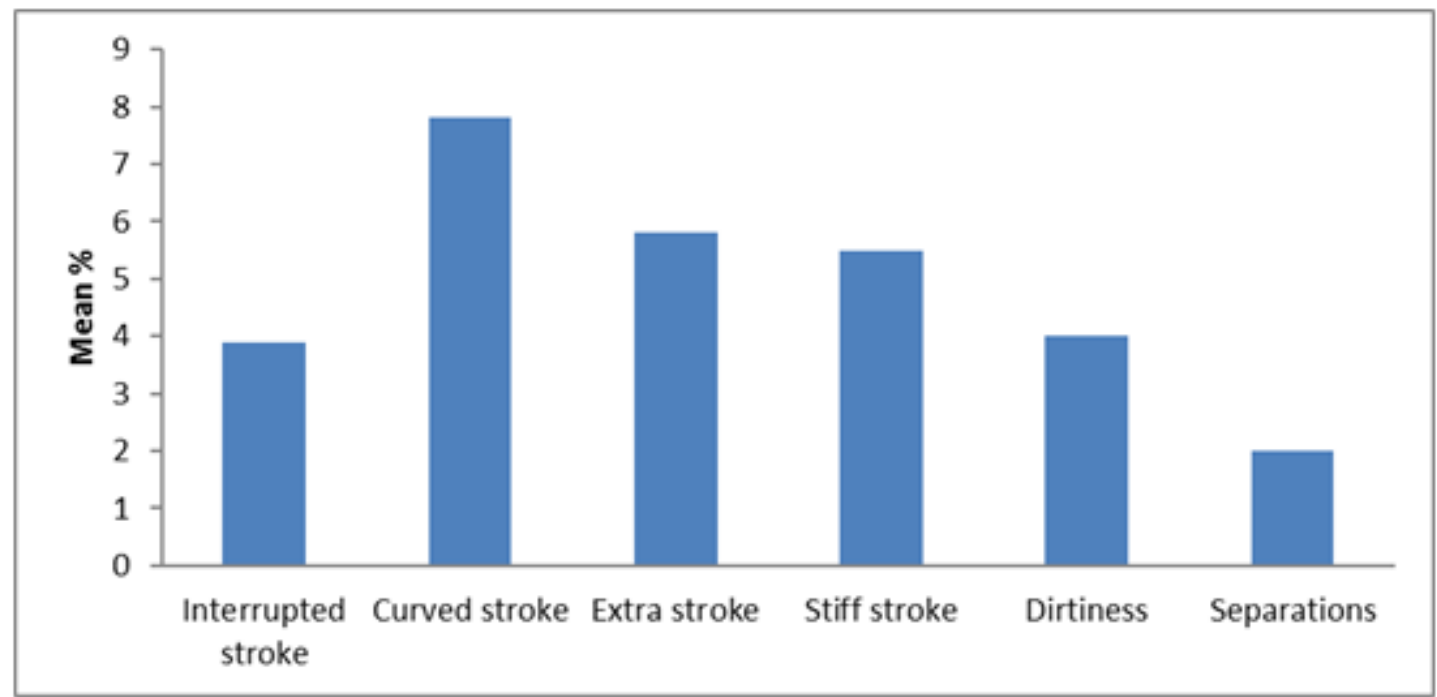

Figure (7): Column chart shows different types of handwriting strokes, dirtiness, inter letters \& inter word spacing and overwriting among the studied patients with chronic renal failure and hemodialysis $(n=37)$.

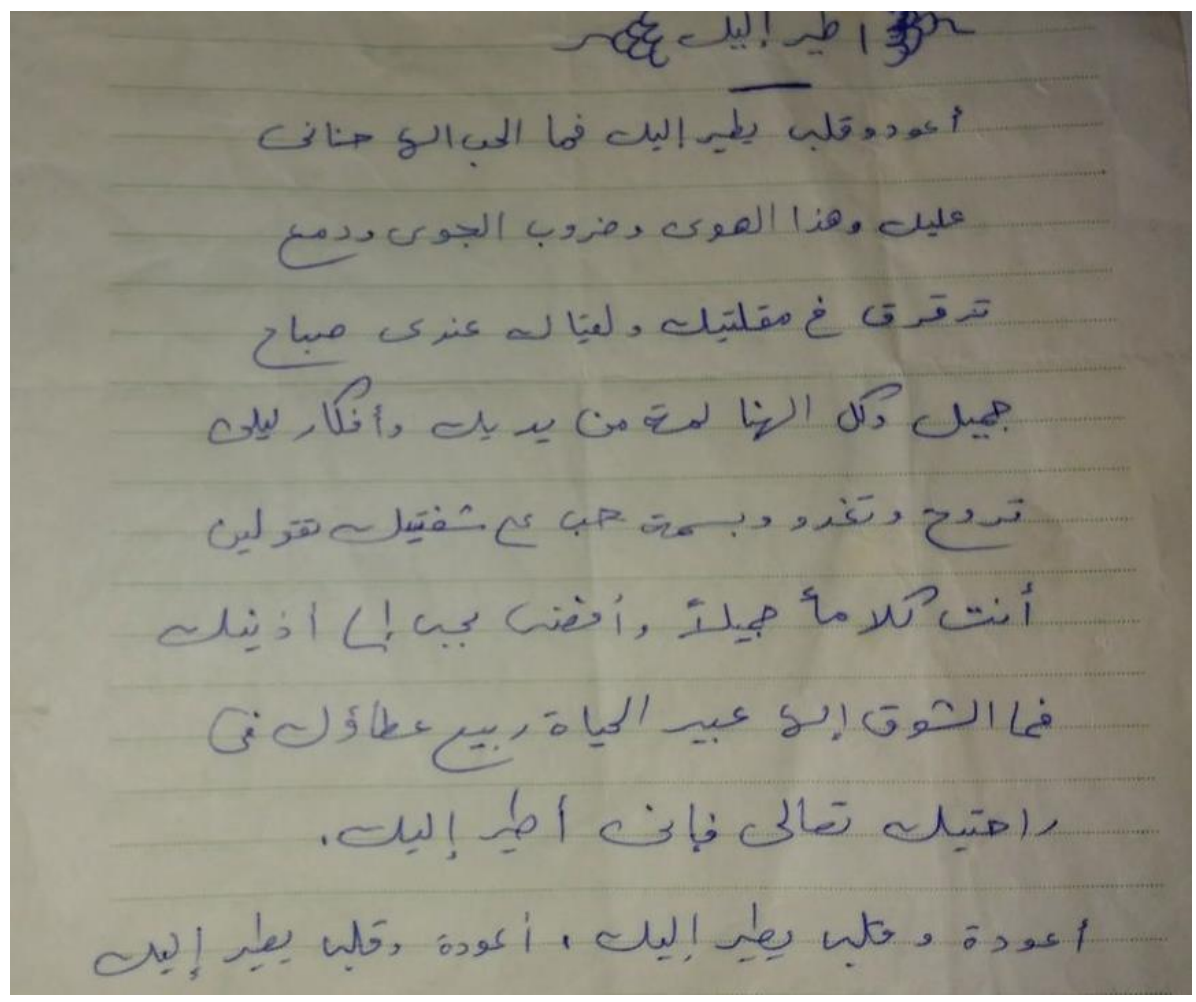

Photograph (2): showing HW of a medium-level educated 34-year-female HD patient recruited from private dialysis center before disease. 


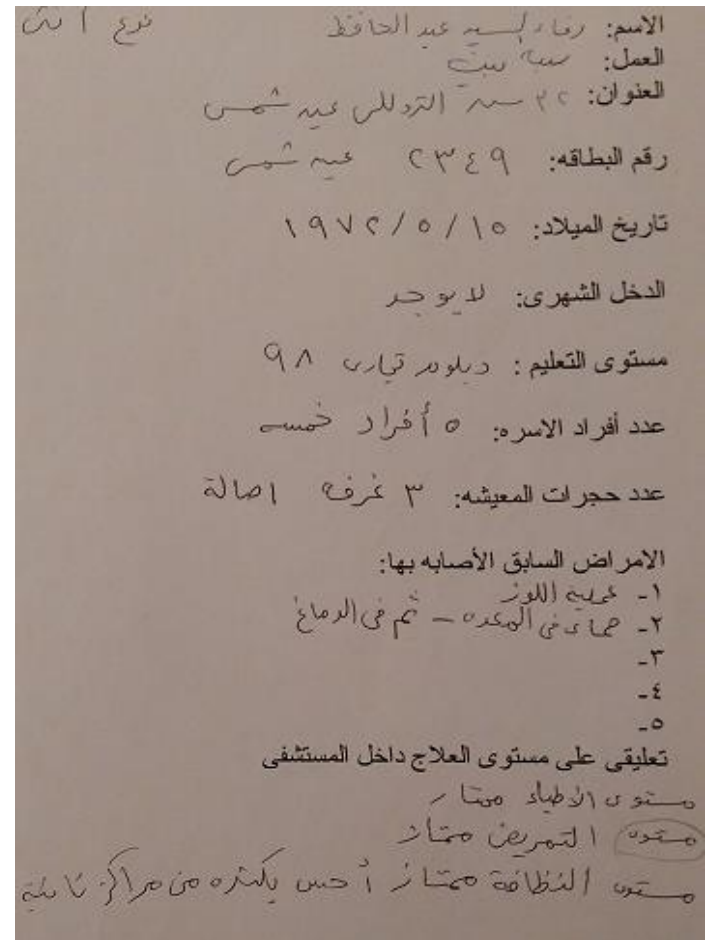

Photograph (3): showing HW of a medium-level educated the same patient after dialysis.

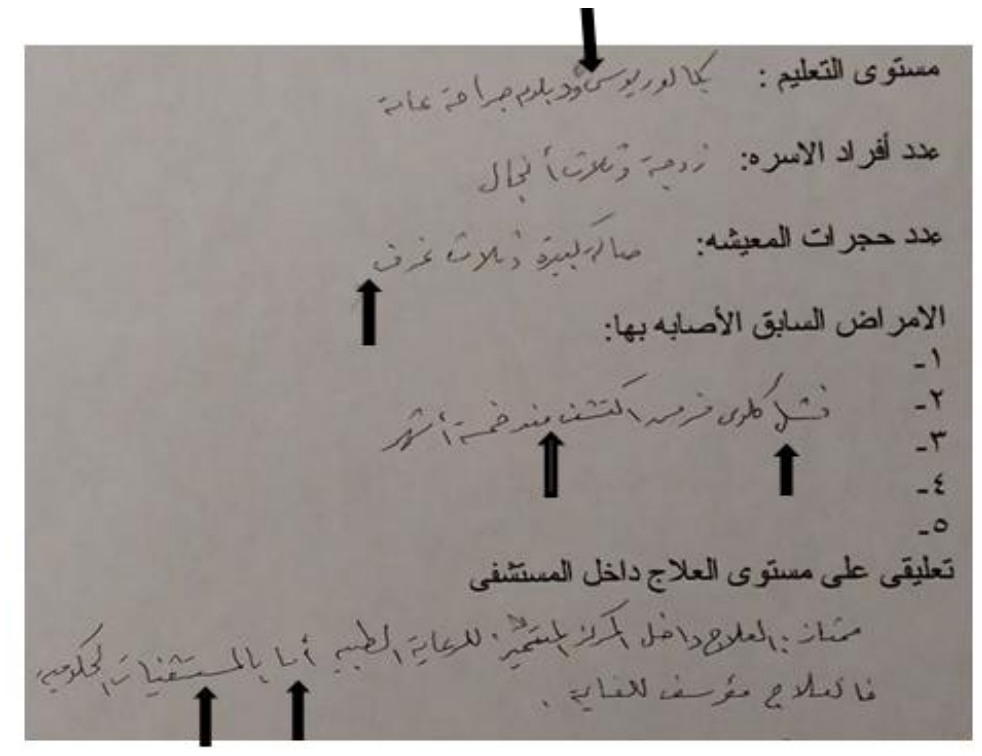

Photograph (4): showing HW of a post-level graduated 51year-male HD patients with semi-tremor recruited from private dialysis center.

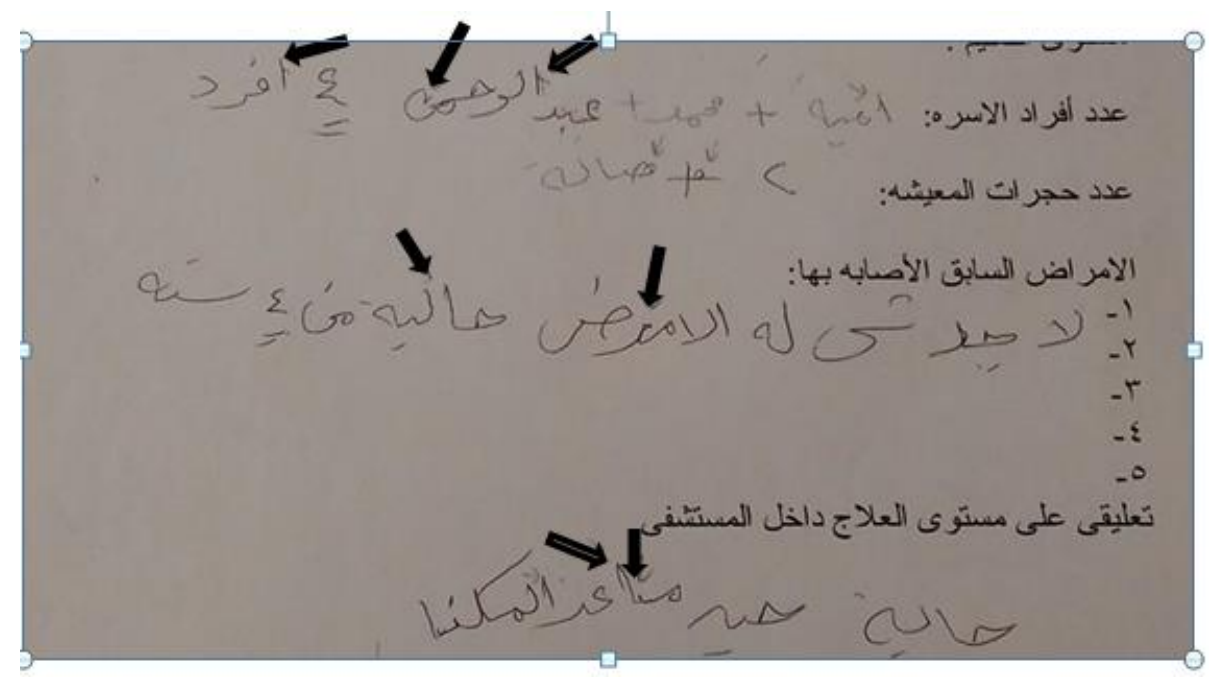

Photograph (5): showing HW of a below-medium graduated 51-year-male HD patient with additional strokes recruited from Nasser institute. 


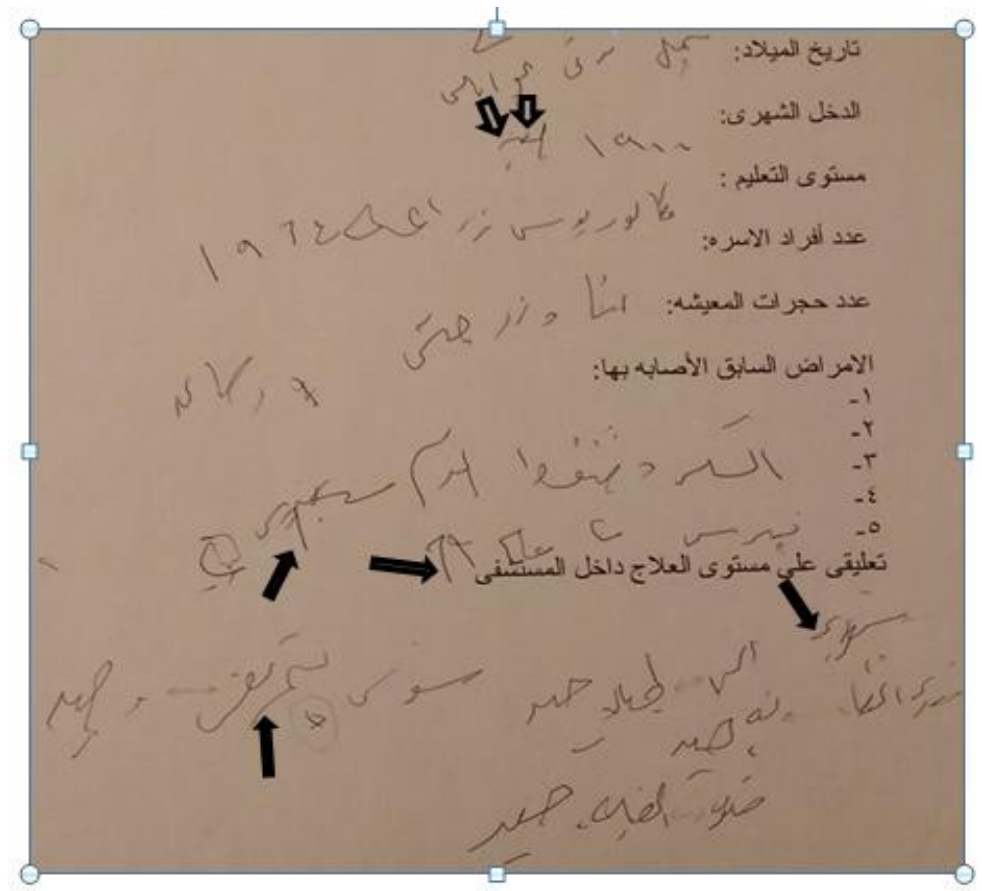

Photograph (6): Showing HW of high-level graduated 42-yearmale HD patient with rigid strokes recruited from private dialysis center.

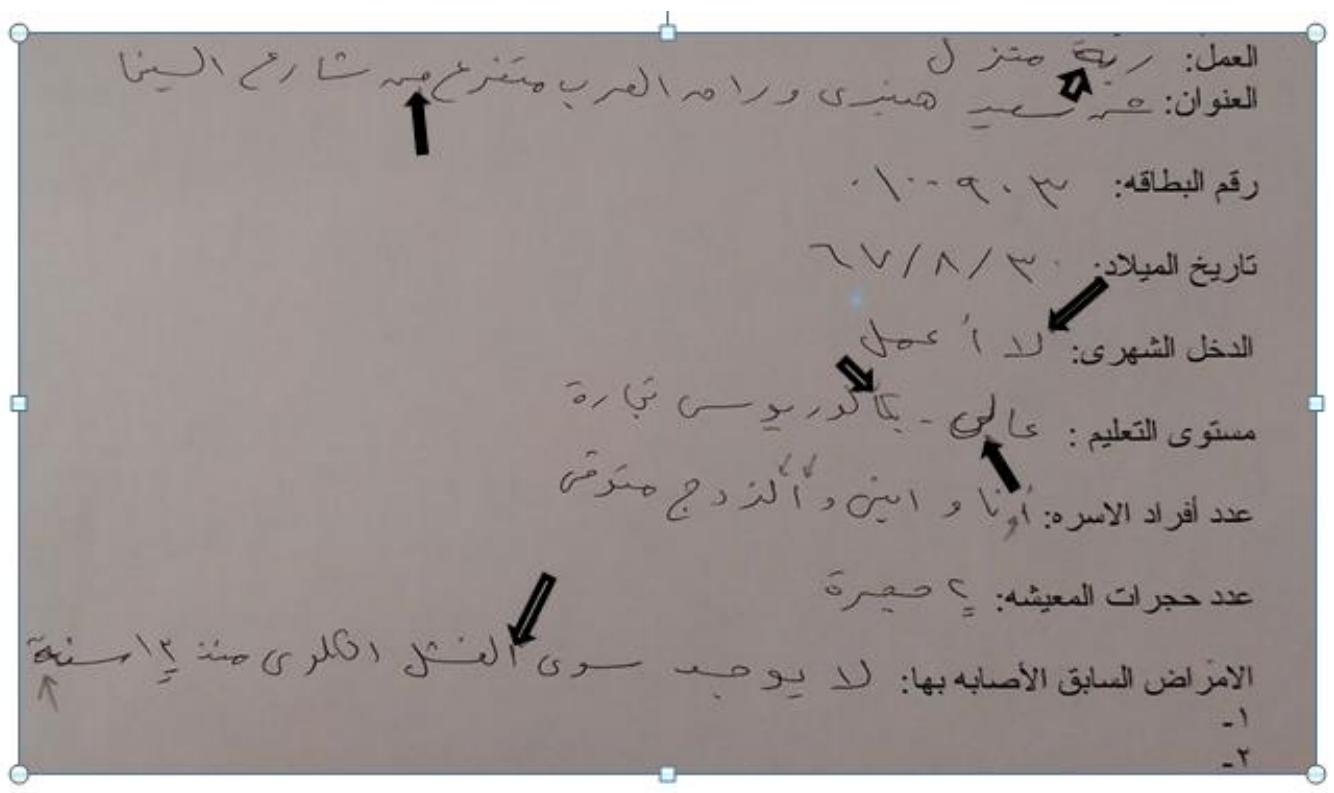

Photograph (7): Showing HW of high-level graduated 40-year-male HD patient with overwriting recruited from private dialysis center. 


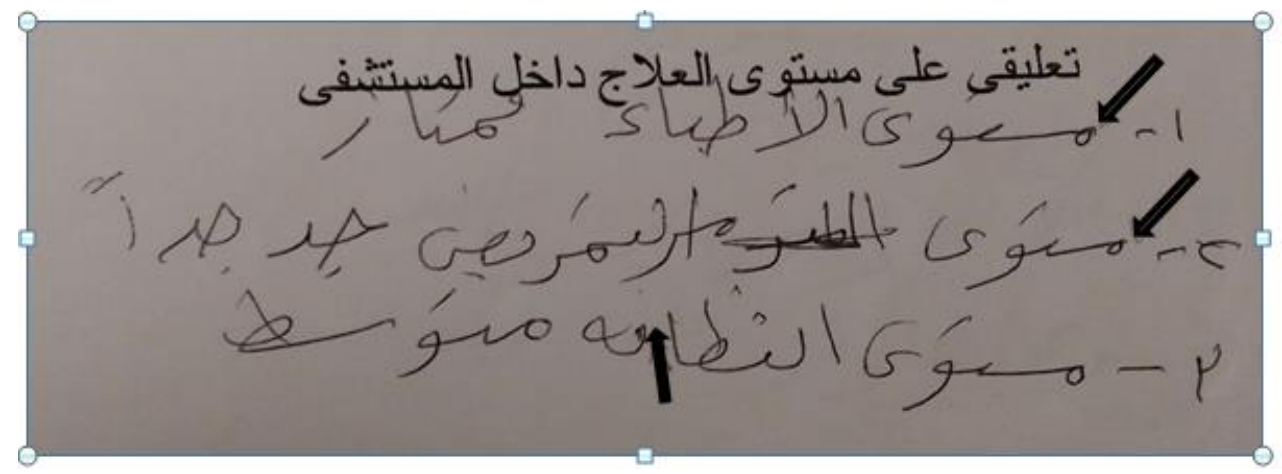

Photograph (8): Showing HW of medium-level graduated74-year-female HD patient with aberration recruited from private dialysis center .

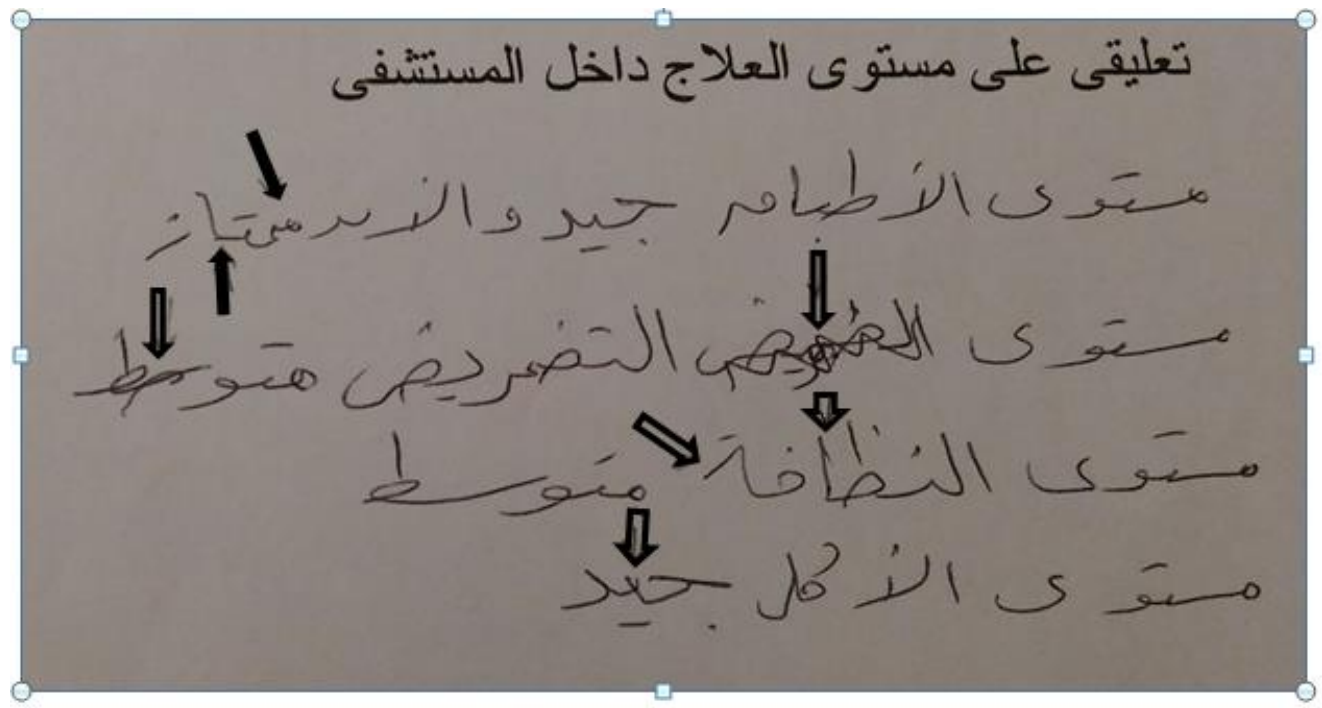

Photograph (9): Showing HW of medium-level graduated 40-year-male HD patient with aberration recruited from Nasser institute .

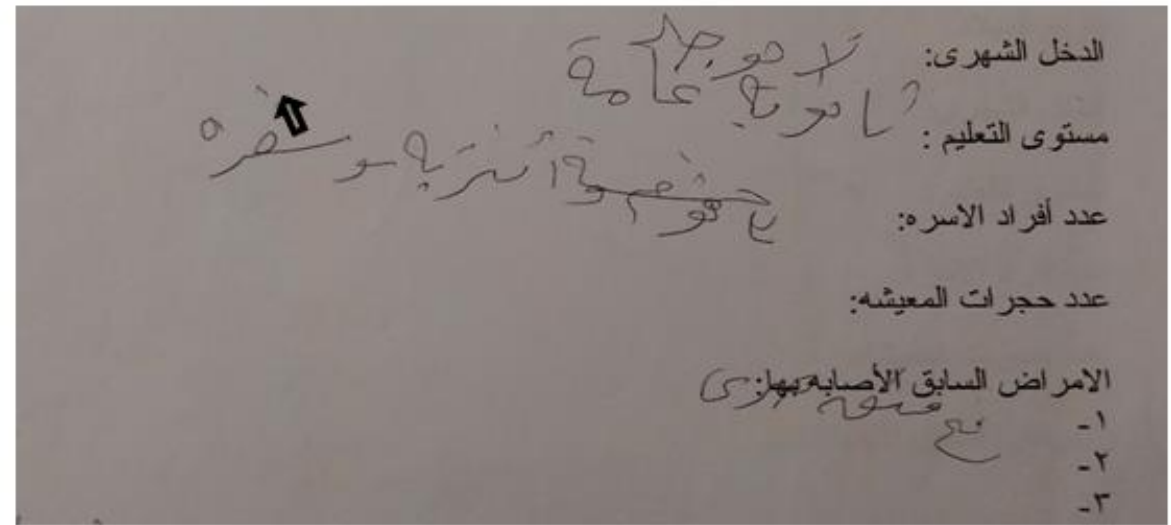

Photograph (10): Showing HW of medium-level graduated 49-yearfemale HD patient with aberration recruited from Nasser institute. 


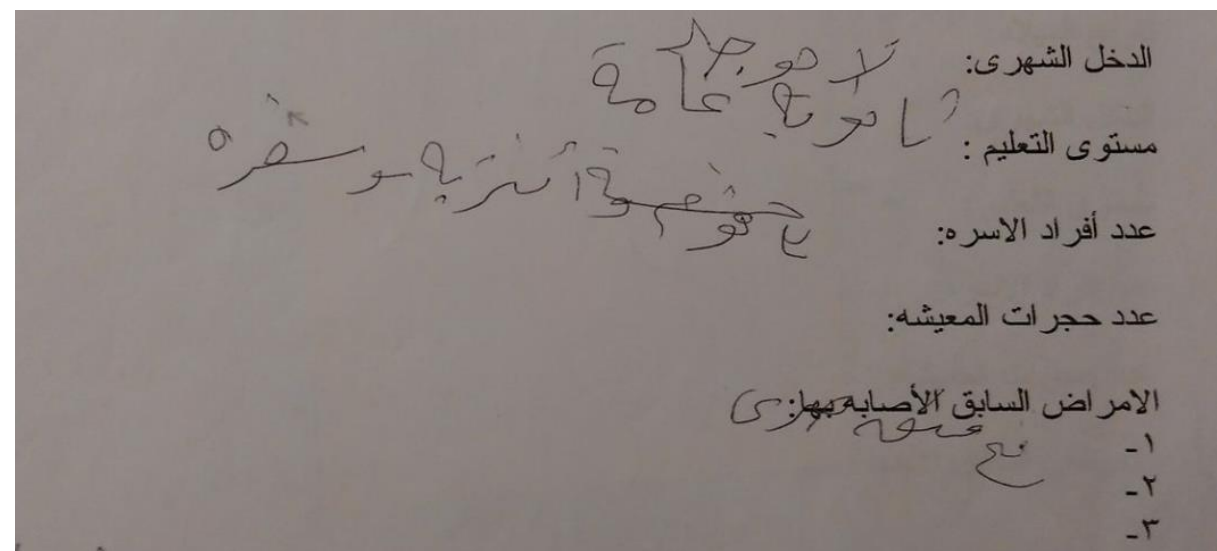

Photograph (11): Showing HW of medium-level graduated 74-year-female HD patient with interference of lines recruited from private dialysis center .

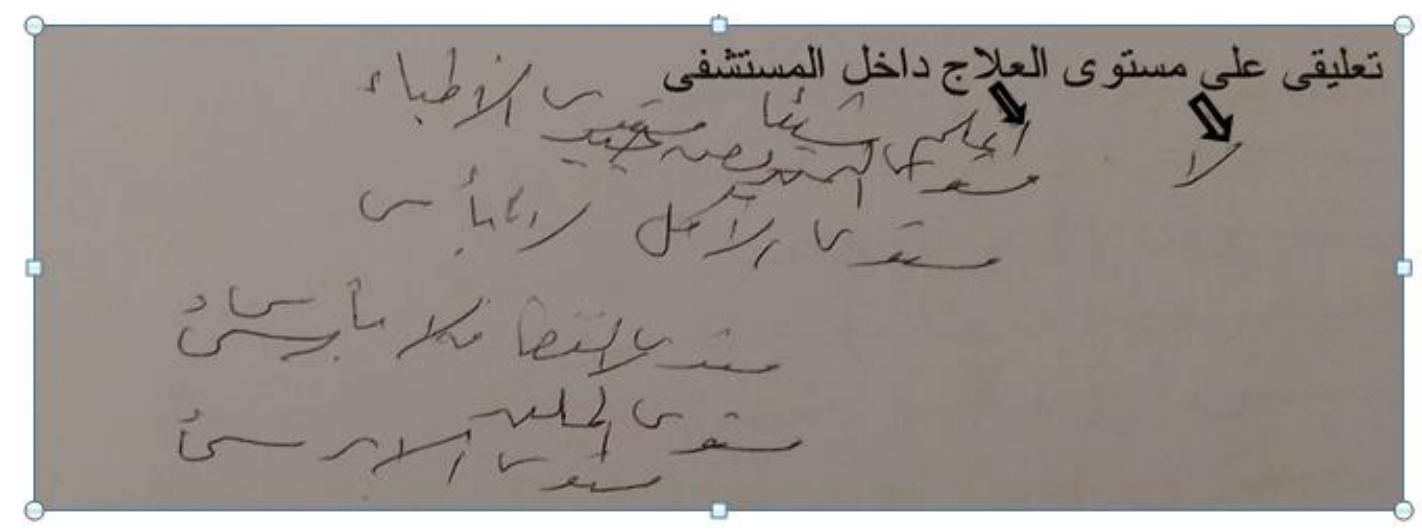

Photograph (12): Showing HW of medium-level graduated 75-year-male HD patient with interference of lines recruited from Nasser institute.

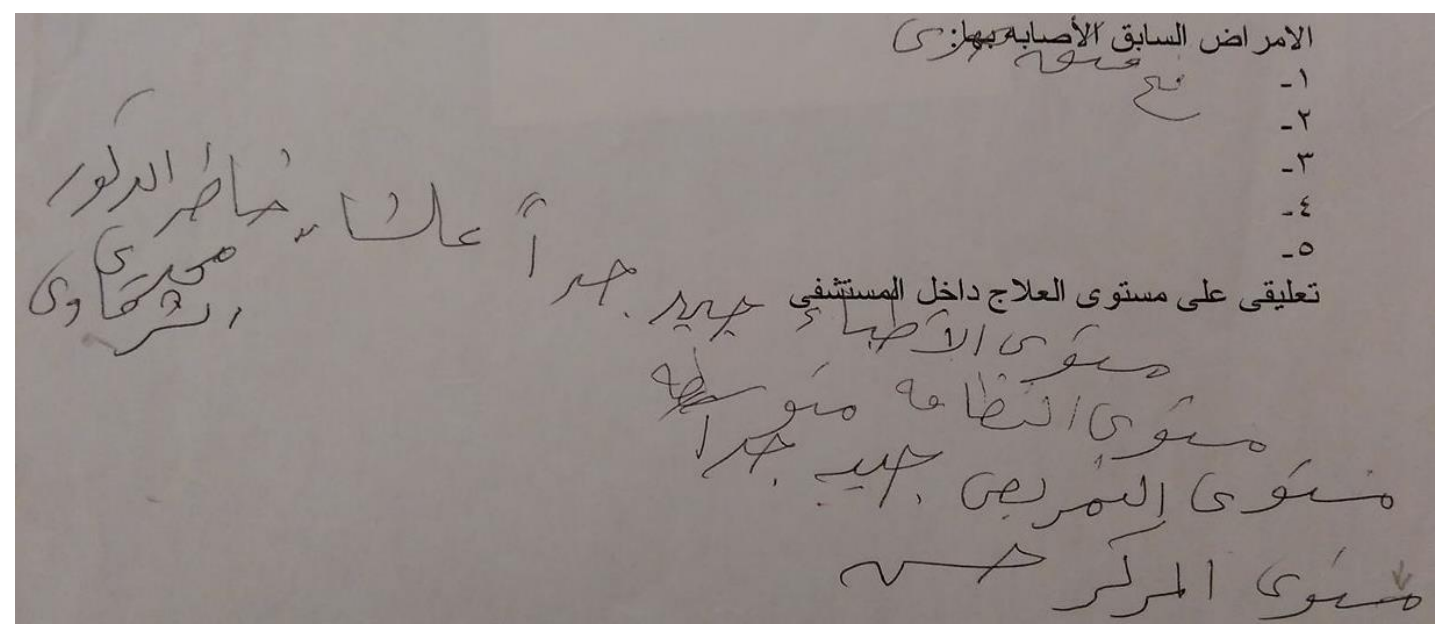

Photograph (13): Showing HW of medium-level graduated 74-year-female HD patient with ascending alignment recruited from private dialysis center . 


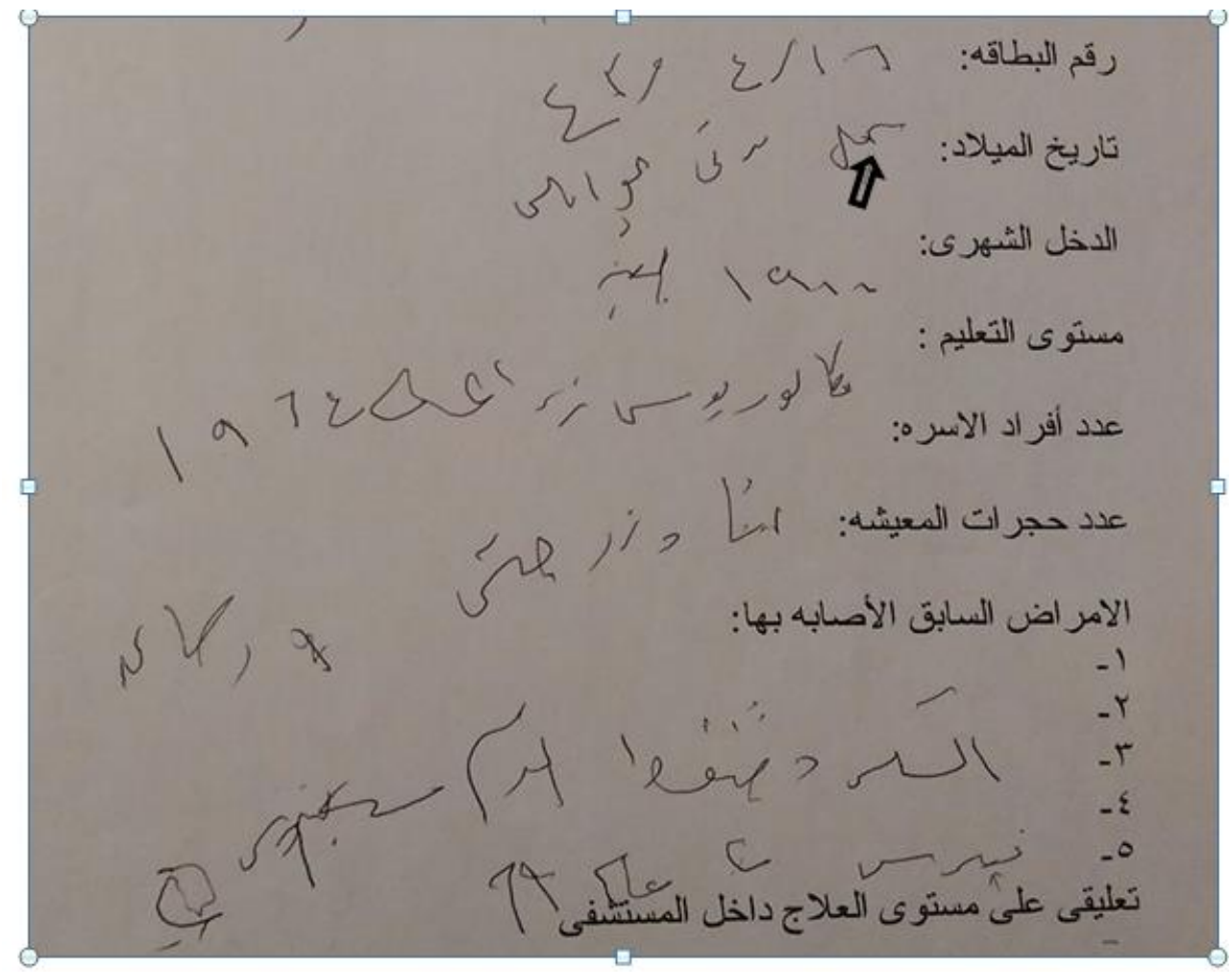

\section{Photograph (14): Showing HW of high-level graduated 63-year-male HD patient with descending alignment recruited from private dialysis center .}

\section{Discussion}

Recently, the relation between chronic kidney disease and neurological disorders with cognitive impairment such as Alzheimer's disease, has been highlighted. This cerebrorenal interaction is considered to be based on small vessel disease. Cerebral and glomerular small vessel disease might have a common soil of pathogenesis, as these organs are closely connected with each other through anatomic and vasoregulatory similarities. ${ }^{22}$

Handwriting implies complex coordinative patterns, in which several motor control subsystems, such as frontal and parietal cortical regions, cerebellar pathways as well as basal ganglia are involved. ${ }^{23}$ The dysfunction of one or several of these subsystems may result in handwriting deficits. On the other hand, recent data have shown that temporal and frontal cortical regions as well as cerebellar control pathways are also involved in control of handwriting movements. ${ }^{24,25 \& 26}$

In the present study, there was a statistically significant difference between the different speeds of handwriting. Slow speed showed the highest percentage of the studied sample (81.1\%). Basalah, (2001b) ${ }^{27}$ reported that mean speed handwriting is the average rate of pen movements which succeed in performing letters and sentences or enough for achievement of writing the whole text, also it may be defined as the number of letter or words which could be written per a unit of time.

Concerning pen pressure grade in the studied sample, there was a statistically significant difference between the different pen pressure grades. Light pressure on the back of the paper which showed the highest prevalence among the studied subjects (62.2\%). Pen Pressure in handwriting shows the physical and mental levels. Heavy pressure indicates commitment and taking things seriously, while light pressure shows sensitivity to atmosphere and also empathy to others. ${ }^{28}$ The pen pressure information contains much individuality of writers $^{29}$ and it is often employed for writer identification. ${ }^{30} \quad$ Hypotonia is an acute symptom of cerebellar damage and may be indicated by reduced pen pressures. $^{31}$ The above mentioned result could be explained ad chronic renal failure manifested with proximal myopathy lead to deterioration of handwriting. ${ }^{18}$

In the present study, there was a statistically significant difference between the different word size grades. Subjects with large word size showed the highest prevalence (46\%), while subjects with small word size showed the least prevalence (13.5\%). The word size can be employed in two ways, either to describe whether writing as a whole is large or small in its formation, in a secondary sense, it may be applied in drawing 
comparisons of the relative proportions of large letters as contrast with those of smaller formations. The second definition is the only one, which holds interest for the handwriting experts. Furthermore, there are national variations even in absolute size of the basic letters. ${ }^{32} \quad$ Van Gemmert et aI., (1998\&2001) ${ }^{33 \& 34}$ observed that there is a significant influence of mental load on micrographia and increased processing demands within the writing task contribute to reduction in writing size. Micrographia has been defined as an impairment of a fine motor skill manifesting mainly as a progressive reduction in amplitude during a writing task.$^{35}$

In this study, semi-tremor, rigid and additional strokes were common types of handwriting stroke in patients with chronic renal failure and haemodialysis. Also $78.4 \%$ of the studied sample showed dirtiness, 97.3\% showed inter letters \& inter and word spacing and 73\% showed over-writing in their handwriting. Letter spacing in handwritten documents represents the openness of sentiment and consequently of the openness of intelligence. Similar to letter spacing word spacing also indicates the disposition towards criticism, and towards argument. It adds to person's personality other than baseline. ${ }^{28}$ However Moon, (1997) ${ }^{36}$ stated that inter-word spacing carried out is a clear index to the writer's mental caliber. It signifies spacing in which the intervals are clear enough for perfect legibility. The untidy spacing proceeds from two or three causes: (a) confused thinking and lack of order in one's mental processes (writing is brain controlled) (b) lack of powers of clear expression or (c) lack of sufficient convenience to others. Also, Chokroverty et al., (1976) ${ }^{37}$ studied 11 cases with sub acutely progressive dialytic encephalopathy patients who had been on haemodialysis for 14 to 36 months was characterized by dementia, language disorder, myoclonic jerks, behavioral disturbance. His writing showed displayed perseveration, hesitancy, omission, and misspelling of words.

Kurella Tamura et aI., (2008) ${ }^{38}$ reported that impaired kidney function was associated with dementia and cognitive impairment. Dialysis dementia is a neurologic disease characterized by speech disturbances, involuntary motor phenomena, seizures and features of Alzheimer.s disease. The current view of its pathogenesis is that it represents a form of aluminum intoxication. ${ }^{39}$ Menichelli et aI., (2008) $)^{40}$ exhibited a severe impairment in writing letters and words in patient with frontotemporal dementia. Handwriting analysis is a novel approach that adds to the tools that the forensic psychiatrist may use in evaluating the validity of a deceased person's last will, or indeed of any controversial document written by hand. It should be used judiciously, taking into account the full spectrum of the available data and evidence. The writing of Alzheimer's demented people contain omission of letters, repetition of letters, and improper connection of words, yet show very little loss in writing skill as would normally be expected. As the disease progresses all patients eventually lose the ability to write, although at what point in the progression of events this occurs will vary with each writer. ${ }^{41}$

Cognitive impairment is highly prevalent among hemodialysis patients and is associated with increased morbidity and mortality. A higher prevalence of non- memory impairment may suggest an underlying vascular versus neurodegenerative mechanism. ${ }^{42}$ Balestrino et al., $(2012)^{43}$ showed that handwriting is compromised in cognitive impairment and that the degree of writing impairment is related to the severity of cognitive impairment. Silveri et aI., (2007) $)^{44}$ found a correlation between the degree of cognitive impairment and spelling mistakes.

A positive influence of mood on handwriting performance, Mergl et aI., (2004) ${ }^{45}$ revealed that depressed patients wrote slower than healthy controls. It may be argued that handwriting is a highly automated skill and that it is therefore unlikely that motor dysfunction in patients with depression affects this skill in a considerable amount. Thus handwriting performance as everyday activity resulting from an optimal cooperation of the manual sensorimotor network rather than from a selective activation of a single part of this network. Fontana et aI., (2008) $)^{46}$ provided an objective way to analyze handwriting, and to test its reliability and correlation with mental deterioration. Handwriting may give clues to the cognitive status of the writer, thus its analysis might be helpful, for example, in the forensic evaluation of a controversial handwritten last will. The handwriting of a person with motor function degradation will be generally less consistent across repetitions of the same word than will the handwriting of healthy people. This lack of consistency, which is related to legibility, increases with tremor. Symptoms such as tremor, and the consequent degradation of fine motor skills, have important implications for the field of forensic document examination. For example, a forensic expert may be asked to determine whether a document has been written by a person who suffers from a neurological disease. This could be critical if there are questions about the authenticity of a document that is claimed to have been written by someone suffering from a motor dysfunction such as tremor. Alternatively, there may be a dispute over whether a document had been written before or after the onset of the disease. In this situation, experts need empirical evidence to guide them as to how handwriting changes in the presence of neurological 
disturbances. Furthermore, it is essential to ascertain the differential influence of stress on the movement characteristics of healthy people, and of those with motor function degradation. ${ }^{47}$

In conclusion, because handwriting is arranged mentally and performed neuro- muscularly; hemodialysis patients had a characteristic impairment in handwriting skills which may indicate degree of cognitive deficit that cannot be elicited by clinical examination and investigations. These changes may carry great medico legal implications, and should be considered when analyzing their handwriting. So the handwriting examiner when asked to examine the handwriting of a hemodialysis patient must have some knowledge about the disease and put considerations of how renal failure can affect handwriting skills.

\section{References}

Thomassen AJ.W.M. and Teulings H.L. Time, size, and shape in handwriting: Exploring spatio-temporal relationships at different levels. In Michon J. A. , \& Jackson J. B. (Eds.) Time, mind, and behavior(1985): pp. 253-263. Heidelberg: Springer-Verlag.

Van-Vugt P. , Paquier P. , Kees L. Increased writing activity in neurological conditions: a re-view and clinical study Neurol. Neurosurg .. 61 (5) (1985): pp. 519- 514.

Reinders-Messelink H.A. , Schoemaker M.M. , Hofte M. , Goeken L.N.H. , Kingma A., van den Briel M.M. , Kamps W.A. Fine motor and handwriting problems after treatment for childhood acute lymphoblastic leukemia Medical and Pediatric Oncology,27(6)_ (1996): pp.551555.

Judie W. Handwriting changes due to aging and Parkinson's syndrome. Forensic Science International, 88(1997): pp. 197-21.

Jin H., Yan' Susan R., Paul M., Rachelle S.D., Hong L. Alzheimer's disease and mild cognitive impairment deteriorate fine movement control. Journal of Psychiatric Research, 42(14) (2008): pp. 1203-1212

Beck J. Handwriting of the Alcoholic Forensic Sci Int., 28(1) (1985): pp. 19-26.

Phillips J.G. Characteristics of handwriting of patients with Huntington's disease Movement Disorder, 9(5) (1994): pp. 521-30.

Mauro Verrelli. Chronic renal failure Nephro Sci, 231(2004): pp. 2- 8.

Mahoney C.A., and Arieff Uremic encephalopathies A.I., Clinical, biochemical and experimental features Am J Kidney Dis, 2(1982): pp. 324336.

De Deyn P.P., Saxena V. K., Abts H., Borggreve F., D'Hooge R., Marescau B. Clinical and pathophysiological aspects of neurological complications in renal failure Acta Neurol Belg, 92(4)(1992): pp.191-206.

Dunea G.Dialysis dementia: an epidemic that came and went ASAIOJ, 47(3) (2001): pp. 192-4.

Fukunishi I., Kitaoka T., Shirai T., Kino K., Kanematsu E., Sato Y. Psychiatric disorders among patients undergoing hemodialysis therapy Nephron, 91(2) (2002): pp. 344-7.

Savazzi G.M., Cusmano F., Vinci S., Allegri L. Progression of cerebal atrophy in patients on regular hemodialysis treatment: long-term follow-up with cerebral computed tomography Nephron, 69(1) (1995): pp. 29 -33

Massey E.W., Bowman M.H., Rozear M.P. Asterixis mimicking drop attacks in chronic renal failure Neurology, 38(4)(1988): pp. 663.

1Artieda, Muruzabal J., Larumbe R., Garcia de Casasola C., lA.Obeso Cortical mechanisms mediating asterixis Mov Disord, 7(3) (1992): pp. 209-16.

Chadwick D. and French A. T. Uraemic myoclonus: an example of reticular reflex myoclonus? J Neurol Neurosurg Psychiatry, 42(1) (1979): pp. 52-5.

1 Aminoff M. Uremic twitch convulsive syndrome. In: Neurology and general medicine Raskin NH, editor. Philadelphia: Churchill Livingstone, (1995): p. 303-19.

Campistol 1. M. Uremic myopathy Kidney International, 62(2002): pp. 1901-1913.

Theofilou P.Depression and anxiety in patients with chronic renal failure: The effect of sociodemographic characteristics International Jo urnal of Nephrology, 11(2011): pp. 6

Murray A. M., Tupper D.E., Knopman D. S., Gilbertson D.T., Pederson S.L., Li S., Smith G. E., Hochhalter A. K. , A, Collins J. , Kane R. L. Cognitive impairment in hemodialysis patients is common. Neurology, 69(1)(2007): pp.120

Basalah R. F. Handwriting analysis In: Limitation of Scientific Evidence in Questioned Document Cases. Nobar Printing House, Cairo, Egypt (2001): p. 566-603.

Mogi M. and Horiuchi M. Clinical interaction between brain and kidney in small vessel disease Cardiology Research and Practice,(2011): pp.5

Katanoda K., Yoshikawa K., Sugishita M. A functional MRI study on the neural substrates for writing Hum Brain Mapp, 13(2001): pp. 34--42.

Eichhorn T.E. , Gsser T. , Mai N. , Marquardt C. , Arnold G. , Schwarz J. , Oertel W.H. WH.Computational analysis of open 
loop handwriting movements in Parkinson's disease: a rapid method to detect dopamimetic effects Mov Disord, 11(3) (1996): pp. 289-297.

Gallucci R. M., Phillips J.G. , Bradshaw J.L., Vaddadi K.S. , Pantel C. is Kinematic analysis of handwriting movements in schizophrenic patients Bioi Psychiatry, 41 (1997): pp. 830-833.

Tucha O. , Aschenbrenner S., Eichhammer P. , Putzhamme A. , Sartor H. , Klein H.E., Lange K. W. The impact of tricyclic antidepressants and selective serotonin re- uptake inhibitors on handwriting movements of patients with depression Psychopharmacology (Berl), 159(2002): pp.211-215.

Basalah R.F. Handwriting analysis In: Limitation of Scientific Evidence in Questioned Document Cases. Nobar Printing House, Cairo, Egypt(200 1b): p.575-578.

Prasad S. , Singh V.K. , Sapre A. Handwriting Analysis based on Segmentation Method for Prediction of Human Personality using Support Vector Machine International Journal of Computer Applications, 8(12) (2010): pp. 25-29.

Yamaki Y., Mizutani Y., Komatsu N. Extraction of personal features from stroke shape, writing pressure and pen inclination in ordinary characters Proc. ICDAR, 99(1999): pp. 426-429.

Kikuchi M. and Akamatsu N. Development of speedy and high sensitive pen pressure and writer identification Proe. ICDAR, 01 (2001): pp.1040- 1044.

Diener H. C. and Dichgans 1. Pathophysiology of cerebellar ataxia Movement Disorder, 7(1992): pp. 95-109.

Miller 1.T. Writing machines Forensic Science International, 13(1979): pp. 1-14.

Van Gemmert A.W. , Teulings H. L. Stelmach G.E. The influence of mental and motor load on handwriting movements in parkinsonian patients Acta Psycho I (Amst),100(1998): pp. 161-75.

Van Gemmert A.W., Teulings H. L., Stelmach G.E. Parkinsonian patients reduce their stroke size with increased processing demands Brain Cogn, 47(2001): pp. 504-12.

Nakamura M., Hamamoto M., Uchida S. A case of micrographia after subcortical infarction: Possible involvement of frontal lobe function Eur J Neurol, 10(2003): pp. 593-6.
Moon W. H. A survey of handwriting styles by Geographic Location Journal of Forensic Sciences, 22(4) (1997): pp. 827-834.

Chokroverty S., Bruetman M.E., Berger V., Reyes M.G. Progressive dialytic encephalopathy Journal of Neurology, Neurosurgery, and Psychiatry, 39(1976): pp. 411-419.

Kurella Tamura M., Wadley V., Yaffe K. Kidney function and cognitive impairment in US adults: the Reasons for Geographic and Racial Differences in Stroke (REGARDS) Study American Journal of Kidney Diseases, 52(2)(2008): pp.227-234.

Supanc V. , Vargek-Solter V. , Demarin V. Metabolic encephalopathies Acta Clin Croat, 42(2003): pp. 351-357.

Menichelli A., Rapp B. , Semenza C. Allographic agraphia: A case study Cortex, 44(7) (2008): pp. 861-868.

Behrendt IE. Alzheimer's disease and its effect on and writing Journal of Forensic Sciences JFSCA, 29 (1) (1984): pp. 87-91.

Post IB., Morin K.G. , Sano M. , Jegede A.B. , Langhoff E. , Spungen A. M. Increased Presence of Cognitive Impairment in Hemodialysis Patients in the Absence of Neurological Events Am J Nephrol, 35(2012): pp. 120-126.

Balestrino M., Fontana P., Terzuoli S., Volpe S., Inglese M.L. , Cocito L.Altered Handwriting Suggests Cognitive Impairment and May Be Relevant to Posthumous Evaluation J Forensic Sci, 57(5)(2012): pp.1252-1258.

Silveri M.C., Corda F., Di Nardo M. Central and peripheral aspects of writing disorders in Alzheimer's disease $\mathrm{J}$ Clin Exp Neuropsychol, 29(2007): pp. 179-86.

Mergl R. , Juckel G., Rihl r., Hen kel V., Karner M. , Tigges P. , Schroter A., Hegerl U.Kinematical analysis of handwriting movements in depressed patients Acta Psychiatr Scand, 109(2004): pp. 383-391.

Fontana P., Dagnino F., Cocito L., Balestrino M. Handwriting as a gauge of cognitive status: a novel forensic tool for posthumous evaluation of testamentary capacity Neurol Sci, 292 (2008): pp. 57-261.

Longstaff M.O. and R. A. Heath The influence of tremor on handwriting performance under conditions of low and intermediate physical stress Journal of Forensic Document Examination, 11(2000): pp. 25- 44. 


\section{الملخص العزبحى}

\section{دراسة مبدئية لتقيم بعض التغيرات في الكتابة اليدوية لبعض المرضى المصريين المصابين بالفشل الكلوي}

هالة عباس محمد' و مجدى الثرقاوي † و فاطمة أغا؟

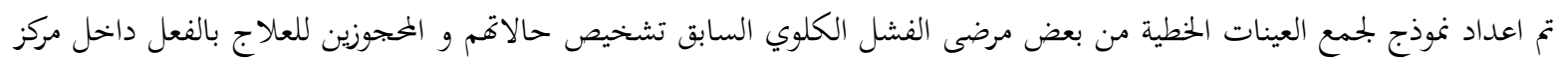

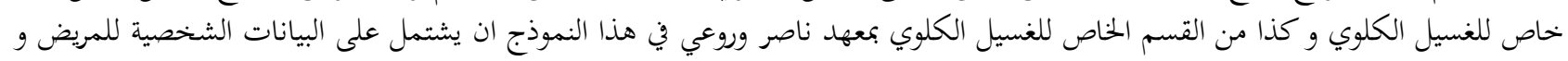

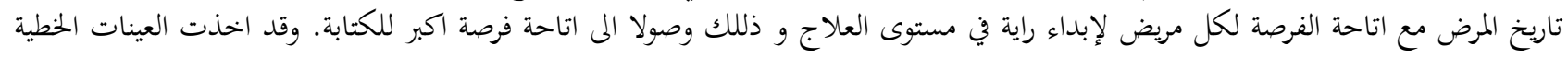

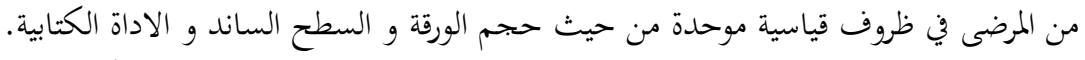

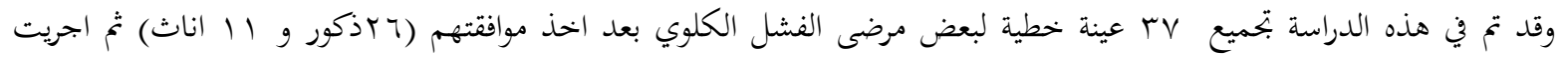

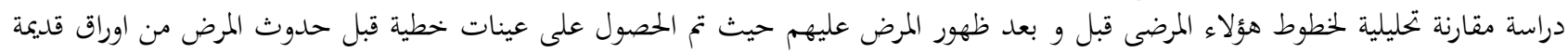

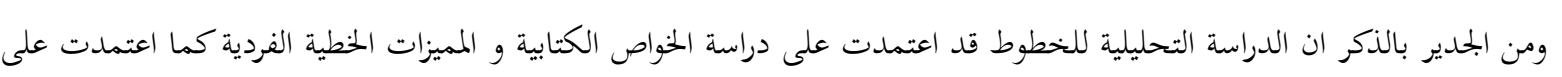

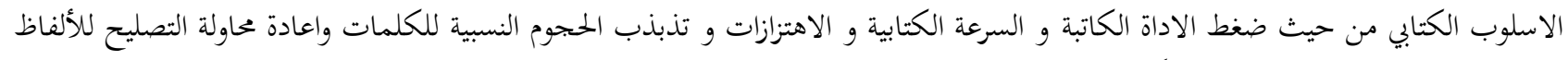

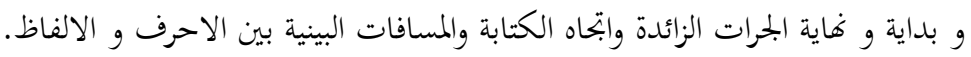

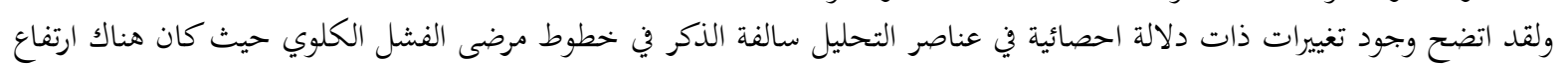

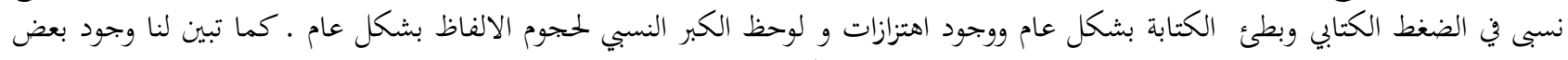

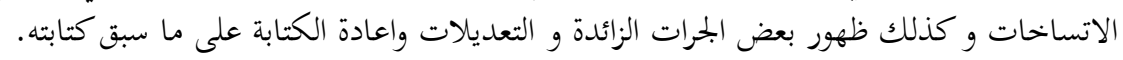

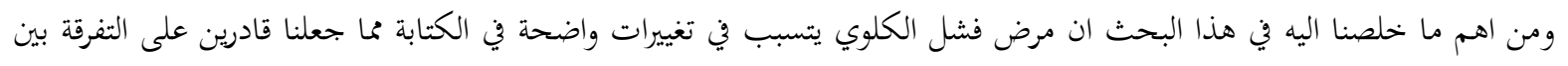

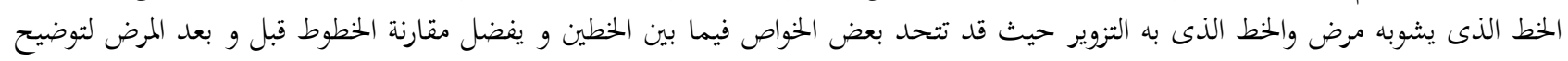

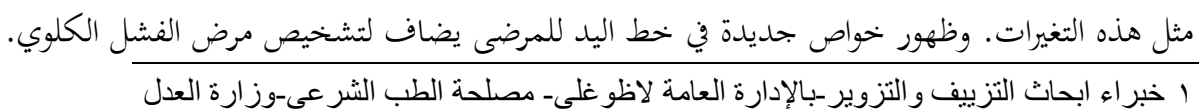

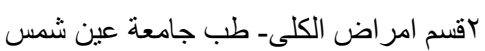

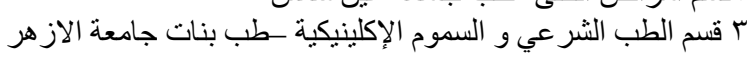

